Suggested Title:

\title{
A Gateway to Understanding Confined Ions
}

If a charged electrode with a flat surface is immersed in a liquid containing ions, it is relatively well understood that the oppositely-charged ions will be attracted to the interface, forming a so-called electrical double layer. However, what happens if the surface of the electrode is not flat but contains nanopores or channels? The answer to this seemingly simple question is surprisingly complex and is not yet fully understood. Moreover, it is central to understanding how important and emerging technologies such as electrochemical supercapacitors function on the molecular level. ${ }^{1}$ Much of what we do know about the behaviour of nanoconfined ions has come from modelling and simulations, ${ }^{2}$ but recently new experimental approaches are starting to provide tantalising insight into real-life systems. ${ }^{3}$ Writing in Nature Nanotechnology, Xiao et al., have demonstrated a new approach based on electrolyte gating which may expand the experimental toolkit and provide an additional lens through which to view the behaviour of nanoconfined ions in real-time. ${ }^{4}$

The technique of electrolyte gating involves putting a semiconductor electrode into contact with an ionic liquid or solution, and applying a gate voltage to set up an electrical double layer at the interface (Fig. 1a). ${ }^{5}$ The accumulation of electrons or holes within the electrode surface increases the conductivity of the semiconductor. Measurement of gating-induced conductance changes has been widely used to study insulator-to-metal transitions, superconductivity, and other electronic phase transitions in materials. The work by Xiao et al. turns electrolyte gating on its head: instead studying the electronic structure of the solid electrode, they set out to see if it could be used to study the behaviour of the ions in the liquid electrolyte.

Part of the challenge with applying electrolyte gating in this context is that the effect is only observed in semiconductors, whereas many model electrode materials are based on graphite-like nanoporous carbon which has metallic conducting properties. However, reduced graphene oxide behaves like a $p$-type semiconductor due to the presence of structural defects. In previous work, the same group has developed an approach for fabricating multi-layered graphene membranes (MGMs) from reduced graphene oxide, in which the interlayer distances between the graphene sheets are controllable from $10 \mathrm{~nm}$ down to subnanometre separations. ${ }^{6}$ The researchers hypothesise that, providing the MGM sheets are sufficiently separated, the electronic structure of the electrode should be more like defective graphene (semiconducting) than graphite (conducting) and the electrolyte gating effect should be observable. Furthermore, since the MGM pore width is tuneable, these materials provide an ideal model system for studying adsorbed ion behaviour.

Using a specially-designed two-electrode cell with an additional platinum contact to measure the trans-membrane conductance (Fig. 1b), Xiao et al. performed a electrochemical measurements on a MGM with $10 \mathrm{~nm}$ layer spacing in an aqueous $\mathrm{KCl}$ electrolyte. As the cell voltage was swept across a range of $\pm 0.8 \mathrm{~V}$, the trans-membrane conductance was found to approximately double. Additional analysis ruled out contributions from redox reactions, electrode expansion or changes in contact resistance, leaving electrolyte gating as the most likely source of the observed results. Further confirmation came from conventional back gating measurements on reduced graphene oxide in a field-effect transistor, where very similar conductance changes were observed.

Having successfully identified electrolyte gating, the next step was to see if this effect is sensitive to the electrolyte ion dynamics. To do this, measurements were performed on a set of MGMs with layer spacings of between $5-0.6 \mathrm{~nm}$. For larger layer spacings, very similar behaviour to the proofof-concept experiment was observed, indicating free movement of ions in and out of the nanochannels during charging (Fig. 1c). Intriguingly, different behaviour was observed for layer spacings below $1.0 \mathrm{~nm}$, particularly when electrolytes containing large molecular ions were used. In these experiments, a marked hysteresis was observed between the cell voltage and trans-membrane 
conductance. Similar results were observed in constant current experiments where the peak conductance was seen several seconds after the ionic current was reversed (Fig. 1d). These observations point towards increased nanoconfinement and reduced ion mobility when the pore width approaches the bare ion diameter - a phenomenon which is particularly pertinent in the field of supercapacitor energy storage devices, where confinement effects have been predicted to yield significant capacitance increases. ${ }^{2}$

The work by Xiao et al. provides a new perspective from which to view the behaviour and dynamics of confined ions, something which is currently of high interest yet notoriously difficult to do. One of the advantages of electrolyte gating is that it is intrinsically a real-time technique and can therefore potentially capture some of the fast timescale processes that are difficult to track with other methods. However, further work will be required to better understand the detailed origins of and the factors influencing - the gating effect, and to determine the resolution with which it can probe the structure of the electrical double layer. Of particular interest is how the arrangement and solvation states of ions within the electrical double layer is affected by the electrode structure and the chemical properties of the electrolyte. If electrolyte gating can help to answer some of these questions it could have a very big impact in the field.

\section{References}

1. Salanne, M. et al. Nature Energy 1, 16070 (2016).

2. Federov, M. V.; Kornyshev, A. A. Chem. Rev. 114, 2978-3036 (2014).

3. Forse, A. C.; Merlet, C.; Griffin, J. M.; Grey. C. P. J. Am. Chem. Soc. 138, 5731-5744 (2016).

4. Xiao, J. et al. Nature Nano.

5. Leighton, C. Nature Mater. 18, 13-18, (2019).

6. Yang, X.; Cheng, C.; Wang, Y.; Qiu, L.; Li, D. Science 341, 534-537 (2013). 
